\title{
Evaluation of LL-37 antimicrobial peptide derivatives alone and in combination with vancomycin against $S$. aureus
}

\author{
James F. Shurko ${ }^{1,2} \cdot$ Ralph S. Galega ${ }^{1} \cdot$ Chuxi Li $^{1} \cdot$ Grace C. Lee $^{1,2}$ \\ Received: 21 May 2018 / Revised: 5 July 2018 / Accepted: 11 July 2018 / Published online: 17 August 2018 \\ (c) The Author(s) 2018. This article is published with open access
}

\begin{abstract}
Treatment of Staphylococcus aureus infections continues to be a challenge due to antimicrobial resistance. Endogenous antimicrobial peptides may offer a new option for treating $S$. aureus infections but several factors limit their clinical utility. Herein, we studied the activity of the antimicrobial peptide LL-37 and two truncated derivatives, LL-13 and LL-17 alone and in combination with vancomycin against a range of drug-resistant $S$. aureus strains including methicillin resistant $S$. aureus (MRSA) and vancomycin resistant S. aureus (VRSA) strains in vitro. When used with vancomycin, LL-13 and LL-17 displayed synergy against VRSA and showed the ability to restore sensitivity to vancomycin after pretreatment. In addition, LL-13 and LL-17 showed a strong ability to inhibit S. aureus biofilm production. LL-37 derivatives may be useful in treating infections that are resistant to vancomycin or in scenarios where biofilm formation is a concern.
\end{abstract}

\section{Description}

Staphylococcus aureus is an important clinical pathogen and is a leading cause of a wide range of infections including bacteremia, infective endocarditis, pneumonia and skin and soft tissue infections [1]. Methicillin resistant $S$. aureus (MRSA) infections account for 11,000 related deaths and $\$ 14.5$ billion in health care costs per year in the USA [2]. Vancomycin is the standard of care for gram positive infections including those caused by MRSA, but its frequent use has led to the development of antimicrobial resistance. Treatment is further complicated by the ability of $S$. aureus to form biofilms which can alter immune response, decrease drug exposure and delay wound healing [3]. These issues highlight the need to develop alternative or adjunctive therapies for the treatment of $S$. aureus infections.

Antimicrobial peptides (AMPs) are a diverse class of naturally occurring molecules that are produced as a first

$\triangle$ Grace C. Lee

Leeg3@uthscsa.edu

1 College of Pharmacy, The University of Texas at Austin, Austin, TX 78712, USA

2 Pharmacotherapy Education and Research Center, School of Medicine, The University of Texas Health San Antonio, San Antonio, TX 78229, USA line of defense by multicellular organisms. AMPs have broad activity to directly kill bacteria, yeasts, fungi, viruses, and cancer cells. Cathelicidins are amphipathic, cationic, AMPs found in humans and other species. Stored in neutrophils, they represent an important part of the innate immune defense. LL-37 is an AMP derived endogenously from the human cathelicidin hCAP18, and has been isolated from several sites including bone marrow, breast milk, salivary glands and the testes [4]. Prior studies have demonstrated that LL-37 exhibits a broad range of activity against gram positive and gram negative organisms and can drive chemotaxis of leukocytes in times of infection $[5,6]$.

LL-37 has been shown to act directly against pathogens through the disruption of cell membranes and inhibition of cell wall, nucleic acid, and protein biosynthesis [7-9]. Several studies have described LL-37's activity against $S$. aureus including preventing biofilm formation [10, 11]. However, a major translational limitation of LL-37 is its limited stability due to cleavage by endogenous enzymes found in the gut (trypsin, pepsin) pancreas (elastace) and serum (plasmin) [12, 13]. Further, enzymes secreted by organisms including S. aureus (aureolysin, V8 protease) have shown the ability to cleave LL-37 [14]. Moreover, LL37 has been associated with non-specific cytotoxicities (e.g., leukocyte, T-cell, and red blood cell viability) $[15,16]$.

Shorter-derivatives of LL-37 can overcome some of these limitations by improving stability, reducing toxicities, and lowering production costs. However, their activity 
against $S$. aureus remains unknown. The aim of this study was to assess the activity of these shorter-derivatives alone and in combination with vancomycin against methicillin susceptible $S$. aureus (MSSA), MRSA, vancomycin intermediate susceptible $S$. aureus (VISA) and vancomycin resistant $S$. aureus (VRSA) strains. Burton and Steel described the helical and amphipathic nature of LL-37 particularly between residues 12 and 29. Since these properties are integral to LL-37's ability to interact with cell membranes, derivatives encompassing residues from this region were selected for this study [17].

Experiments were conducted against ATCC strains 25923 (MSSA), 43300 (MRSA) and 700699 (VISA) and BR-VRSA (VRSA) acquired from BEI resources (Manassas, VA). Vancomycin was acquired from Sigma-Aldrich (St. Louis, MO). The AMP LL-37 (LLGDFFRKSKEKIGKEFKRIVQRIKDFLRNLVPRTES), and derivatives LL-13 (IGKEFKRIVQRIKDFLRNLVPRTES) and LL-17 (FKRIVQRIKDFLR) were synthesized by Anaspec (Fremont, CA). Purity was assessed using high performance liquid chromatography.

Minimum inhibitory concentrations (MICs) for vancomycin against the $S$. aureus isolates were performed in triplicate by broth microdilution as per Clinical and Laboratory Standards Institute guidelines [18]. MICs of AMPs were conducted using concentrations ranging from 0.25 to $1024 \mu \mathrm{g} / \mathrm{mL}$. Serial 2-fold dilutions were performed in 96-well plates prior to adding inoculum. Plates were then incubated at $35^{\circ} \mathrm{C}$ for $24 \mathrm{~h}$ and MICs were determined by observing the lowest concentration that visually inhibited growth.

In addition, the ability of AMPs to resensitize VISA and VRSA to vancomycin was tested by exposing ATCC 700699 and BR-VRSA to LL-13 and LL-17 at $1 / 4$ X MIC for one hour before being added to broth containing 2-fold serial dilutions of vancomycin ranging from 0.125 to 256 $\mu \mathrm{g} / \mathrm{mL}$. Plates were then incubated at $35^{\circ} \mathrm{C}$ for $24 \mathrm{~h}$ and MICs were determined by observing the lowest concentration that visually inhibited growth.

The activity of AMPs used in combination with vancomycin was studied by checkerboard assays conducted in 96well microtiter plates. Plates contained concentrations of vancomycin and a single AMP at multiples of their respective MICs. The fractional inhibitory concentration index (FICI), was estimated using the following equation: (MIC of drug A in combination)/(MIC of drug A alone) + (MIC of drug B in combination)/(MIC of drug B alone) at $24 \mathrm{~h}$ of incubation. Combinations were considered synergistic if the FICI was $\leq 0.5$ and antagonistic if the FICI was $>4$. FICIs between 1 and 4 were classified as indifferent.

Inhibition of $24 \mathrm{~h}$ biofilms formed by ATCC 25923 (a known biofilm producer) was assessed by semiquantitative plate assays, as described by O'toole [19]. Biofilms were formed in 96-well microtiter plates in the presence of vancomycin and AMPs individually at $1 / 2$ and $1 / 4$ X MIC. Biofilms were washed to prevent background staining by planktonic bacteria and stained using crystal violet. Biofilms were dried and remaining dye was solubilized with $30 \%$ acetic acid. After solubilizing, materials were transferred to a clear polystyrene plate and absorbance $\left(\mathrm{OD}_{595}\right)$ was measured using a BioTek plate reader (Winooski, VT).

Overall, the AMPs displayed weak activity against the 4 $S$. aureus strains when used alone (Table 1). However, when LL-13 and LL-17 were used in combination with vancomycin, they displayed synergy against VRSA at concentrations equal to $1 / 8 \mathrm{X}$ MIC. Further, after brief exposure of VRSA to a sub-inhibitory concentration of the peptide derivatives $(128 \mu \mathrm{g} / \mathrm{mL})$, the MIC of vancomycin dropped substantially. The vancomycin MIC was reduced by 128 -fold from $512 \mu \mathrm{g} / \mathrm{mL}$ to $4 \mu \mathrm{g} / \mathrm{mL}$ after exposure to LL-13 and greater than 500-fold from 512 to $1 \mu \mathrm{g} / \mathrm{mL}$ after exposure to LL-17. Notably, vancomycin MICs fell within intermediate and susceptible ranges respectively after pretreatment. In addition, all three AMPs demonstrated the ability to inhibit $S$. aureus biofilm formation when used at concentrations equal to $1 / 4$ and $1 / 2$ X MIC. LL-37 reduced biofilm formation 2-fold at $32 \mu \mathrm{g} / \mathrm{mL}\left(\mathrm{OD}_{595}\right.$ reduced from 1.02 to 0.44 ) and $64 \mu \mathrm{g} / \mathrm{mL}\left(\mathrm{OD}_{595}\right.$ reduced from 1.02 to $0.48)$ respectively. Further, at concentrations of $128 \mu \mathrm{g} / \mathrm{mL}$ and $256 \mu \mathrm{g} / \mathrm{mL}, \mathrm{LL}-13\left(\mathrm{OD}_{595}\right.$ reduced from 1.04 to 0.00 and 0.02 respectively) and LL-17 $\left(\mathrm{OD}_{595}\right.$ reduced from 1.00 to 0.00 and 0.02 , respectively) prevented biofilm formation almost entirely.

Table 1 Fractional inhibitory concentration indices of AMPs used in combination with vancomycin

\begin{tabular}{lllllll}
\hline Strain & AMP & $\begin{array}{l}\text { MIC of } \\
\text { AMP }\end{array}$ & $\begin{array}{l}\text { MIC of } \\
\text { VAN }\end{array}$ & FIC $^{\mathrm{a}}$ & FIC $^{\mathrm{b}}$ & FICI \\
\hline 25923 & LL-13 & 512 & 1 & 0.25 & 0.5 & 0.75 \\
& LL-17 & 512 & 1 & 0.25 & 0.5 & 0.75 \\
& LL-37 & 128 & 1 & 0.25 & 0.25 & $0.5^{\mathrm{c}}$ \\
43300 & LL-13 & 256 & 1 & 0.13 & 0.5 & 0.63 \\
& LL-17 & 512 & 1 & 0.13 & 0.5 & 0.63 \\
700699 & LL-37 & 256 & 1 & 0.5 & 0.5 & 1 \\
& LL-13 & 1024 & 4 & 0.06 & 0.5 & 0.56 \\
& LL-17 & 1024 & 4 & 1 & 1 & 2 \\
& LL-37 & 64 & 4 & 0.25 & 0.5 & 0.75 \\
BR-VRSA & LL-13 & 512 & 512 & 0.13 & 0.25 & $0.38^{\mathrm{c}}$ \\
& LL-17 & 512 & 512 & 0.13 & 0.25 & $0.38^{\mathrm{c}}$ \\
& LL-37 & 64 & 512 & 1 & 1 & 2 \\
\hline
\end{tabular}

$A M P$ antimicrobial peptides, $M I C$ minimum inhibitory concentration $(\mu \mathrm{g} / \mathrm{mL}), V A N$ vancomycin

${ }^{\mathrm{a}} \mathrm{FIC}$ MIC of AMP in combination/MIC of AMP alone

${ }^{\mathrm{b}} \mathrm{FIC}$ MIC of vancomycin in combination/MIC of vancomycin alone ${ }^{\mathrm{c}}$ Indicates synergistic effects 
This study compared the activities of two short chain derivatives, LL-13 and LL-17, of the AMP LL-37 in combination with vancomycin against $S$. aureus strains with varying degrees of resistance and assessed their ability to inhibit biofilm production. Both LL-13 and LL-17 displayed significant synergy when used in combination with vancomycin against VRSA. Further, a short pretreatment with LL-13 and LL-17 at sub-inhibitory concentrations displayed a substantial increase in vancomycin susceptibility against VRSA, and reduced the MIC to susceptible ranges. These results demonstrate LL-13 and LL-17's activity in potentiating vancomycin in $S$. aureus. While further studies must be performed to elucidate the mechanism of synergy, vancomycin has been shown to exhibit greater activity against $S$. aureus including VRSA when used in combination with agents that disrupt cell walls [20]. Mohamed et al. theorized that de novo membrane disrupting AMPs may display synergy with vancomycin through increased drug exposure at the cell's division septum where vancomycin exerts activity. In their study, permeabilization was demonstrated by leakage of calcein dye from preloaded cells. In cells treated with AMPs, 80\% of calcein dye leaked from cells after one hour, whereas untreated cells did not leak dye. Further, when using bipody labeled vancomycin, fluorescence was increased at the septum after pretreatment with sub therapeutic concentrations of AMPs. In their study, the combination of AMPs and vancomycin displayed synergy $(\mathrm{FICI}=0.27)$ against VRSA and pretreatment of VRSA with AMPs reduced vancomycin MICs 2-512 fold [20]. The increase in vancomycin susceptibility may support alternative treatment paradigms using AMPs to restore sensitivity to vancomycin in patients that have developed resistance.

Another major concern in treating S. aureus infections is the formation of biofilms. LL-13 and LL-17 displayed remarkable ability to inhibit biofilm production, and nearly prevented biofilm formation completely, whereas LL-37 reduced formation by half. While this study evaluated the ability of AMPs to prevent biofilm formation, other studies have demonstrated LL-37's potential to eradicate previously formed biofilms. Using a similar assay, Haisma et al. [11] demonstrated a four-fold reduction of biofilm formation by LL-37 at concentrations up to $32 \mu \mathrm{M}$. Further, the investigators demonstrated a concentration dependent degradation of established biofilms. Mature biofilms ( $24 \mathrm{~h}$ growth) were exposed to LL-37 for $4 \mathrm{~h}$ before assessing viable bacteria. In their study viable bacteria was reduced by $\sim 25 \%$ at concentrations above $12 \mu \mathrm{M}$. The short derivatives showed a distinct ability to prevent biofilm formation and together these studies suggest the potential of LL-37 derivatives in treating patients where biofilms may be present including skin and soft tissue infections, bone and joint infections, endocarditis, and device-related infections. Further studies assessing the eradication of formed biofilms should be further explored.

Overall, LL-17 and LL-13 displayed synergistic activity against multidrug-resistant $S$. aureus strains, reversing VRSA MICs into susceptible ranges, and demonstrated the ability to prevent biofilm production. These agents show potential for treating $S$. aureus infections when vancomycin resistance and biofilm production are concerns.

\section{Compliance with ethical standards}

Conflict of interest The authors declare that they have no conflict of interest.

Open Access This article is licensed under a Creative Commons Attribution 4.0 International License, which permits use, sharing, adaptation, distribution and reproduction in any medium or format, as long as you give appropriate credit to the original author(s) and the source, provide a link to the Creative Commons license, and indicate if changes were made. The images or other third party material in this article are included in the article's Creative Commons license, unless indicated otherwise in a credit line to the material. If material is not included in the article's Creative Commons license and your intended use is not permitted by statutory regulation or exceeds the permitted use, you will need to obtain permission directly from the copyright holder. To view a copy of this license, visit http://creativecommons. org/licenses/by/4.0/.

\section{References}

1. Tonga SYC, Davis JS, Eichenbergerb E, Hollandb TL, Fowler VG. Staphylococcus aureus infections: epidemiology, pathophysiology, clinical manifestations, and management. Clin Microbiol Rev. 2015;28:603-61.

2. Centers for Disease Control and Prevention. Antibiotic resistance threats in the United States, 2013. www.cdc.gov/drugresistance/ threat-report-2013/.Accessed 2017.

3. Wolcott RD, et al. Chronic wounds and the medical biofilm paradigm. J Wound Care. 2010;19:45-6, 48-50, 52-3.

4. Dürr UHN, Sudheendra US, Ramamoorthy A. LL-37, the only human member of the cathelicidin family of antimicrobial peptides. Biochim Biophys Acta. 2006;1758:1408-25.

5. Schmidtchen A, Frick IM, Andersson E, Tapper H, Björck L. Proteinases of common pathogenic bacteria degrade and inactivate the antibacterial peptide LL-37. Mol Microbiol. 2002;46:157-68.

6. Yang D, et al. LL-37, the neutrophil granule- and epithelial cell-derived cathelicidin, utilizes formyl peptide receptor-like 1 (FPRL1) as a receptor to chemoattract human peripheral blood neutrophils, monocytes, and T cells. J Exp Med. 2000; 192:1069-74.

7. Zanetti M. Cathelicidins, multifunctional peptides of the innate immunity. J Leukoc Biol. 2004;75:39-48.

8. Yeaman MR, Yount NY. Mechanisms of antimicrobial peptide action and resistance. Pharmacol Rev. 2003;55:27-55.

9. Brogden KA. Antimicrobial peptides: pore formers or metabolic inhibitors in bacteria? Nat Rev Microbiol. 2005;3:238-50.

10. Noore J, Noore A, Li B. Cationic antimicrobial peptide LL-37 is effective against both extra- and intracellular Staphylococcus aureus. Antimicrob Agents Chemother. 2013;57:1283-90. 
11. Haisma ME, et al. LL-37-derived peptides eradicate multidrugresistant Staphylococcus aureus from thermally wounded human skin equivalents. Antimicrob Agents Chemother. 2014; 50:4411-9.

12. Moncla BJ, Prykek K, Rohan LC, Graebing PW. Degradation of naturally occurring and engineered antimicrobial peptides by proteases. Adv Biosci Biotechnol. 2011;2:404-8.

13. Hollands A, et al. A bacterial pathogen co-opts host plasmin to resist killing by cathelicidin antimicrobial peptides. J Biol Chem. 2012;287:40891-7.

14. Sieprawska-Lupa M, et al. Degradation of human antimicrobial peptide LL-37 by Staphylococcus aureus-derived proteinases. Antimicrob Agents Chemother. 2004;48:4673-9.

15. Oren Z, Lerman JC, Gudmundsson GH, Agerberth B, Shai Y. Structure and organization of the human antimicrobial peptide LL-37 in phospholipid membranes: relevance to the molecular basis for its non-cell-selective activity. Biochem J. 1999; 341:501-13.

16. Johansson J, Gudmundsson GH, Rottenberg ME, Berndt KD, Agerberth A. Conformation-dependent antibacterial activity of the naturally occurring human peptide LL-37. J Biol Chem. 1998; 273:3718-24.

17. Burton MF, Steel PG. The chemistry and biology of LL-37. Nat Prod Rep. 2009;12:1574-82.

18. Clinical and Laboratory Standards Institute. Performance standards for antimicrobial susceptibility testing: twenty-fourth informational supplement M100-S24. Wayne, PA: CLSI; 2014.

19. O'toole GA. Microtiter dish biofilm formation assay. J Vis Exp. 2011;47:2437.

20. Mohamed MF, Abdelkhalek A, Seleem MN. Evaluation of short synthetic antimicrobial peptides for treatment of drug-resistant and intracellular Staphylococcus aureus. Sci Rep. 2016;6:29707. 